\title{
COYUNTURAS CRÍTICAS DE UN DESASTRE: EL CASO DEL 27F
}

\author{
Robert L. FunK (rofunk@iap.uchile.cl) \\ Pedro Figueroa (pfiguero@uchile.cl) \\ Instituto de Asuntos Públicos - Universidad de Chile
}

Por medio de la utilización del modelo teórico que ofrece el institucionalismo histórico, el presente artículo tiene como objetivo comprender las reacciones políticas y públicas en las semanas posteriores al terremoto de Chile del 27 de febrero de 2010. Se analiza la centralidad que tiene el rol de la sorpresa en las respuestas a catástrofes. En este sentido, se argumenta que los terremotos son mucho menos parecidos a cualquier otro tipo de desastre natural y mucho más parecidos a un ataque terrorista.

Palabras clave: Terremoto; Institucionalismo histórico; Chile.

\section{CRITICAL JUNCTURES OF A NATURAL DISASTER: THE CASE OF 27F}

Using the theoretical model offered by historical institutionalism, the paper aims to better understand the public and political reactions in the weeks following the Chilean earthquake of 27 February, 2010. Emphasis is placed on surprise as a key factor in disaster response. In this sense, it is argued that earthquakes are in many ways much less like other natural disasters and much more like terrorist attacks.

Keywords: Earthquake; Historical Institutionalism; Chile 


\section{INTRODUCCIÓN}

A comienzos de 2010 Chile se preparaba para una transmisión de mando presidencial, representando un importante paso en el proceso de normalización política emprendida dos décadas antes, y para celebrar un ańo bicentenario. Sin embargo, el último fin de semana de las vacaciones de verano, una inmensa parte del territorio nacional vivió un terremoto que alcanzó 8.8 grados en la escala Richter. A pesar de haber sido uno de los sismos más fuertes de la historia, las normas de construcción, implementadas después de siglos de terremotos y temblores en el territorio nacional, significaron que los daños a la propiedad pudieron ser minimizados. Muchas de las muertes registradas se dieron no por los efectos del terremoto mismo, sino del maremoto que se produjo casi inmediatamente después.

Esto, en parte, resultó en una serie de críticas respecto a la respuesta por parte de las autoridades, tanto en los primeros minutos y horas después de la tragedia como en los días y semanas siguientes. En particular, se cuestionó el papel de la Oficina Nacional de Emergencia (ONEMI) y del Servicio Hidrográfico y Oceanográfico de la Armada (SHOA), y surgieron además cuestionamientos respecto al desempeño de las autoridades militares, políticas y de seguridad.

Un argumento que se utilizó como autodefensa por parte de las autoridades era que el terremoto se produjo durante el último fin de semana de las vacaciones de verano. Implícitamente se postulaba que los organismos encargados de responder no estaban, del punto de vista organizacional o de gestión, mal preparados, sino que simplemente sufrían de un poco feliz pero comprensible estado de "relajo". Sin embargo, como veremos más adelante, las políticas públicas requieren especial atención cuando se trata de fenómenos naturales como los terremotos, en el sentido que tienen que estar bien diseñadas e implementadas a priori. Esto, a la vez, tiene obvias consecuencias con las capacidades de reacción una vez producido el desastre, en cuanto al buen funcionamiento de la institucionalidad, y como consecuencia, un fluido proceso de toma de decisiones. Como se pudo observar en las reacciones políticas y públicas en las semanas posteriores al 27F, este tema es central. 
Para comprender estos procesos y preguntar cuáles son las variables que hacen que los terremotos sean tan distintos de otros desastres naturales, este trabajo utiliza el modelo teórico que nos ofrece el institucionalismo histórico. Veremos la centralidad que tiene el rol de la sorpresa, o impredictibilidad, en las respuestas a catástrofes de este tipo. En este sentido, el artículo postula que los terremotos son mucho menos parecidos a casi cualquier otro tipo de desastre natural, y mucho más parecidos a un ataque terrorista como el que se vivió en los Estados Unidos el 11 de septiembre del 2001.

A continuación se presenta un breve resumen del enfoque de institucionalismo histórico, antes de proceder con una descripción de la literatura sobre desastres naturales. Luego, se aplica el marco teórico a los eventos del 27F.

\section{INSTITUCIONALISMO HISTÓRICO, COYUNTURAS CRÍTICAS Y DESASTRES}

El enfoque del institucionalismo histórico fue desarrollado en Estados Unidos durante las últimas décadas del siglo XX desde el enfoque del denominado "nuevo institucionalismo" (ver, por ejemplo, Peters, 2005). A diferencia del tradicional estudio de las instituciones, analíticamente más descriptivo de las organizaciones, normas y procedimientos formales y su determinación sobre la conducta de los sujetos, el nuevo institucionalismo observa situaciones determinadas por los objetivos y la distribución del poder entre los actores políticos, asumiendo el supuesto de que los factores institucionales determinan de algún modo la conducta de éstos, elaborando a partir de aquello, teorías explicativas de los procesos políticos asociados (Sánchez, 2004: 97).

En tal sentido, el institucionalismo histórico realiza el análisis de la relación entre las instituciones y los agentes políticos y sus funciones desde una perspectiva amplia y comprehensiva. Esta relación es considerada central dentro de la ciencia política debido a que permite avanzar más allá de la descripción del modo en que los factores institucionales (institucionalidad) condicionan, en algún sentido, la capacidad de alterar la agenda y las estrategias de los actores, permitiendo también explicar de qué manera las transformaciones institucionales pueden generar reformulaciones en los objetivos de los propios actores, así como también en las 
ideas e intereses que dan forma a las acciones políticas. Por tanto, el interés de las investigaciones que adoptan este enfoque radica, fundamentalmente, en el conocimiento que aportan acerca de entender cómo instituciones canalizan las demandas y las respuestas políticas, considerando la vinculación proporcional entre conflicto o situaciones críticas, estrategias, acciones y comportamientos políticos (Sánchez, 2004: 97).

Desde distintos puntos de vista, las instituciones pueden ser observadas como el resultado de procesos históricos que se cristalizan en ellas; como símbolos de un legado que se manifiesta, siendo ambas importantes desde la óptica del estudio en relación con su origen más que las funciones que realizan. Por otro lado, los sujetos, contrariamente a la manera en que son visualizados por el institucionalismo racional, son considerados como actores con limitada capacidad en términos de conocimiento, determinados por el cumplimiento o ejecución de las normas (Sánchez, 2004: 98). Pierson y Skocpol (2008) se refieren al institucionalismo histórico como el análisis de las configuraciones organizacionales, centrado en coyunturas críticas y procesos de largo plazo, estableciendo de este modo la comprensión de los fenómenos políticos a partir de contextos amplios y procesos que, por medio de su relación e interacción, reestructuran la política (Skocpol, 2008: 7). A través de esta definición metodológica, el institucionalismo histórico ha logrado acumular un extenso y preciso corpus de conocimiento empírico fundado en la perspectiva de la explicación causal en ámbitos tales como el de las transiciones a la democracia, la relación entre política nacional e internacional, el auge y declive de los autoritarismos, el impacto de la identificación social en política, factores y efectos de los movimientos sociales, en especial, sobre las revoluciones y la evolución de los estados de bienestar y modelos económicos. En este artículo, pretendemos extender el alcance de estas explicaciones a las políticas adoptadas a partir de catástrofes como el terremoto ocurrido en Chile el 27 de febrero de 2010.

\subsection{Aspectos centrales del institucionalismo histórico}

El marco del institucionalismo histórico considera los procesos históricos, definidos a la luz de la búsqueda de causalidad en las explicaciones sobre las variaciones políticas particulares, en relación al análisis de un proceso en el tiempo. En 
este sentido, la extensión del marco temporal de la investigación permite recabar mayores datos a la vez que sensibilizar a los investigadores con las limitantes temporales sobre las que actúan las variables asociadas, obteniendo, finalmente, consideraciones generales acerca de la dimensión histórica de la causalidad (Skocpol, 2008: 12).

Los procesos históricos, entendidos como un factor de análisis, contemplan también dos principios teóricos centrales. El primero de ellos tiene que ver con la idea de asumir los procesos causales como de lento devenir. Se parte de la premisa de que los fenómenos políticos requieren ciertos períodos de tiempo para producirse y desarrollarse en pleno y que sus causas son incrementales solo en el largo plazo. El resultado social es interpretado finalmente como una derivación de las transformaciones sucedidas en varios períodos y como la suma de la aparición de un conjunto de factores causales claves, por lo que el interés del institucionalismo histórico está en conocer las (pre)condiciones estructurales presentes más -incluso- que la temporalidad de los propios acontecimientos. La no referencia a la propiedad más bien pausada de la dimensión social puede inducir al error en explicaciones centradas en elementos desencadenadores más que en las causas de fondo. El segundo factor seńalado considera la historia como un proceso, desde la óptica de la comparación, que hace plausible el enfoque debido a su perspectiva de análisis macroscópica sobre instituciones, organizaciones y tendencias sociales, en relación con los límites del conductismo y la teoría de la elección racional (Skocpol, 2008: 16-18).

Además de los procesos históricos como elemento propio de este enfoque, Pierson y Skocpol señalan la importancia de analizar las instituciones en términos contextuales. En este sentido, se cuestiona el valor de las motivaciones individuales en las causas que son ejecutadas en las dimensiones institucionales y organizacionales, potenciando, por el contrario, la importancia de los efectos de la correspondencia entre estas últimas. En relación con lo anterior, se plantea que los efectos de largo plazo derivados de las decisiones institucionales son productos de procesos sociales, en el marco de lo cual, las instituciones presentan una fuerte capacidad de resistencia frente a los cambios en las condicionantes sociales generales (como resultado del legado político generado por las tendencias institucionales en desarrollo). 
En suma, el institucionalismo histórico concentra la explicación de la estructura social y política asociada a los cambios institucionales en clave de largo plazo, siendo esta una relación que moldea la arena política y a las propias instituciones. Sus teorías causales se posicionan, principalmente, sobre el desenvolvimiento político y económico entre unidades de análisis (como países, regiones o ciudades), influenciadas por la variable independiente representada por instituciones como la burocracia, el electorado y las conexiones entre gobierno y privados (Araújo, 2007). La atención radica, finalmente, en el análisis de los resultados que esas relaciones determinan sobre la vinculación entre estado y sociedad y en el proceso de elaboración de políticas.

\subsection{Path Dependence}

Así como el institucionalismo histórico se enmarca en la escuela del nuevo institucionalismo, la teoría de path dependence (o de trayectorias dependientes) forma parte de este primer enfoque. Se postula que existen situaciones azarosas, ajenas al control de los actores e instituciones, las que, no obstante, adoptan un esquema y función que las determina como path único, es decir, como camino o tendencia dominante que configura una pauta prevaleciente, a la cual los actores deben ajustar sus estrategias.

El desarrollo político, desde esta teoría, contempla la existencia de coyunturas críticas (critical junctures), es decir, momentos claves de crisis de las instituciones, los que, dependiendo de su evolución, por un lado, conducen a las sociedades por distintas trayectorias de desarrollo y, por otro, pueden conformar transformaciones institucionales adaptadas a las condiciones contextuales en relación con las tendencias generales derivadas de la coyuntura. Es por lo tanto, también, un elemento central en el análisis de estos momentos críticos el factor temporal, no solo en referencia al período en que estos ocurren, sino que en estrecha vinculación con la secuencia que adoptan los procesos.

La pieza clave en la explicación de acuerdo a la óptica de la path dependence es el funcionamiento de una retroalimentación positiva (positive feedback) en torno a una determinada acción, la formación de políticas o sus procesos deliberativos (proceso de toma de decisiones). Esta idea señala que el establecimiento de una trayectoria en el esquema político se tiende a robustecer con el paso del tiempo. El 
fundamento de esta aseveración se centra en la existencia de beneficios asociados a la adopción de la pauta política en marcha, en contraste con los costes (crecientes) relativos al abandono de ésta.

En el sentido de lo descrito hasta ahora, los procesos de trayectoria dependiente se conforman a través de dos etapas fundamentales: los efectos derivados de una coyuntura crítica y los mecanismos de retroalimentación asociados a éstos que contribuyen a la repetición de una tendencia en el tiempo. Si bien, los procesos pueden verse afectados de modo importante en sus inicios por perturbaciones menores, una vez que se adopta una tendencia específica resulta difícil que los actores puedan revertir el curso que ésta implica, por lo que los eventos que generan las coyunturas críticas son, bajo este criterio, transcendentales (Pierson y Skocpol, 2008).

En particular, la mencionada dinámica creciente de los beneficios (o de rendimientos) es una justificación central para centrar la atención del análisis en la dimensión temporal de los procesos políticos y las secuencias asociadas. Los teóricos de este enfoque señalan que en la investigación comparada sobre varios países, el orden, comprendido como tiempo y secuencia, de los sucesos ocurridos es determinante para los patrones de desarrollo político. En palabras de Pierson y Skocpol,

la temporización relativa a la secuencia es importante porque los procesos de retroalimentación subsecuentes, que tienen lugar en el tiempo, durante la vida política y social, transforman las consecuencias de desarrollo ulteriores (Pierson y Skocpol, 2008: 14).

La relevancia de factores explicados a partir de la referencia al "por qué" y el "cuándo" se relaciona, además, con el carácter irreversible de ciertas políticas posibles que son marginadas en el proceso de la dinámica creciente de los beneficios.

\subsection{El estudio de "Coyunturas Críticas"}

En lo que respecta al análisis específico sobre las coyunturas críticas (critical junctures), Collier y Collier (1991) las utilizan para identificar patrones de desarrollo a lo largo del tiempo en diferentes países en clave comparada. A su vez, las 
definen como períodos de cambios significativos que comúnmente ocurren de distinta manera en todos los países (o en otras unidades de análisis) y que generan diferentes tipos de legados. Los autores señalan, además, que los momentos críticos pueden implicar un período relativo en el que se adopta una determinada dirección (trayectoria o path) y, por lo general, sus efectos se encuentran entrelazados con otros procesos de cambio. Debido a su enfoque, esta perspectiva es central para el estudio de las crisis, secuencias y frecuencias de desarrollo, continuidad y cambio en políticas económicas nacionales e internacionales, formas de institucionalización (particularmente en clave neo-institucional), entre otros eventos. $\mathrm{O}$, como en este caso planteamos, también puede ser utilizada para evaluar las respuestas en materia de política pública a las catástrofes naturales (Collier y Collier, 1991: 27-28).

\section{Cuadro 1 \\ Path Dependence y Coyunturas Críticas}

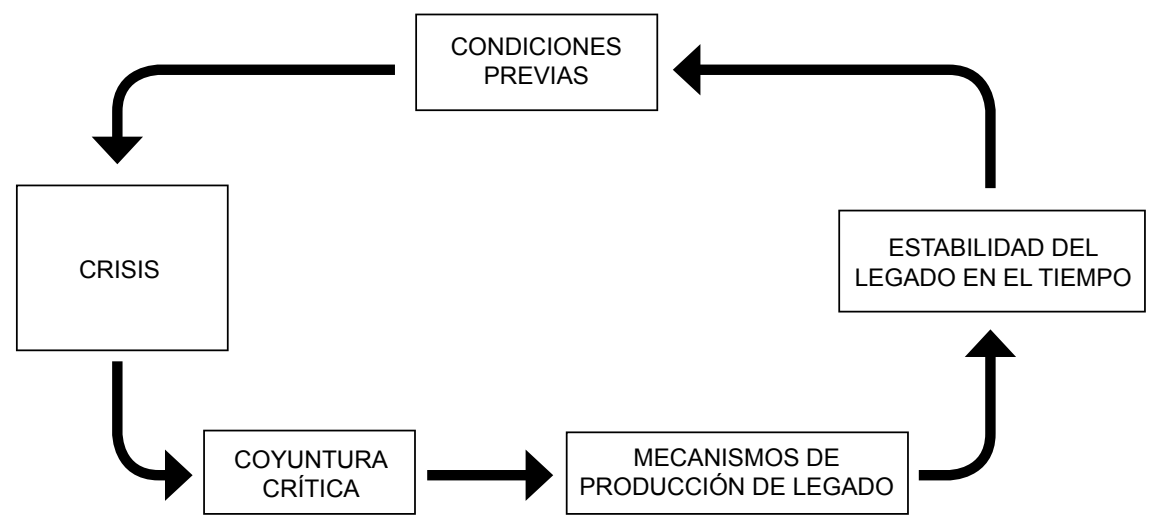

Fuente: Elaboración propia basado en Collier y Collier (1991).

Como demuestra el cuadro 1, el institucionalismo histórico sirve para realizar un rastreo de los momentos claves (coyunturas críticas) que transforman el statu quo en una nueva realidad, a través de las decisiones y respuestas que dan los individuos, influidos por las instituciones. Así, vemos como tanto las personas como las instituciones contribuyen al desarrollo de una nueva realidad o statu quo. 
En particular, la comprensión del enfoque de las coyunturas críticas contempla tres elementos basales: la efectiva presencia de un cambio significativo en cada caso; la afirmación de que este cambio se produjo de formas distintas en cada uno de los casos y la generación de una hipótesis que explique sus consecuencias (Collier y Collier, 1991: 30). Además, existen cuatro puntos posteriores que complementan la teoría. Primero, las condiciones antecedentes, es decir, aquellos factores sobre los cuales se evalúan los momentos críticos y sus legados. En segundo lugar, las crisis derivadas de las condiciones iniciales y desencadenantes de la coyuntura. Los componentes del legado, en tercer lugar, se refieren a sus mecanismos de producción (sobre la idea de que el legado no se concreta de una vez sino que requiere de una serie de etapas), mecanismos de reproducción (se trata de la perpetuación del legado por medio de los procesos institucionales y políticos actuales), y la estabilidad de sus atributos principales. En cuarto lugar, explicaciones rivales que involucran causas constantes. Por último, el deterioro eventual del legado (Collier y Collier, 1991: 30-31).

En un análisis detenido del caso de estudio planteado, cabe considerar los tipos de cambios políticos discontinuos que marginan los antiguos patrones institucionales, en especial, acerca de los aspectos asociados a la resistencia de los esquemas afectos por las coyunturas. En tal sentido cabe considerar, por ejemplo, los problemas suscitados en el gobierno saliente al momento de considerar decisiones como enviar tropas a las zonas afectadas.

En relación al aspecto estrictamente metodológico, podemos señalar que a diferencia de los estudios cuantitativos, donde se considera significativo los hallazgos que explican en parte las variaciones de las condiciones iniciales, en análisis histórico-comparado, el criterio de significancia en las explicaciones contempla que los casos analizados posean características similares en el sentido de comprender que sus patrones son básicamente parecidos durantes periodos de momento crítico. En tal sentido, sostenemos que son comparables los casos del terremoto de 2010 en Chile con el atentado terrorista ocurrido en EE.UU el 11 de septiembre de 2001, debido a un factor esencial de imprevisibilidad de los eventos, distinto a otros casos similares, pero de naturaleza distinta, como por ejemplo, el Huracán Katrina, que pese a ser un desastre natural igual que un terremoto, es en principio "pronosticable". 


\section{ACERCA DEL CONCEPTO DE DESASTRE}

Existen distintos tipos de fenómenos asociados a cada uno de los elementos de la naturaleza. Algunos de estos eventos ocurren de manera periódica y regular, como las lluvias en determinadas zonas geográficas durante épocas específicas del año, mientras que otros, como los terremotos, acaecen en forma irregular sin ningún tipo de patrón que permita pronosticar, a la manera que otros fenómenos, su ocurrencia.

Algunos fenómenos de la naturaleza se constituyen en "desastres", en tanto ocasionan pérdidas de vidas humanas y materiales, entre otros efectos perjudiciales; no obstante, en estricto rigor, el concepto "desastre natural" resulta ser inexacto o, por lo menos vago, toda vez que el adjetivo "natural" hace referencia al fenómeno que origina el desastre y no a los efectos que este pudiera producir, aun cuando éstos podrían estar relacionados con el entorno donde ocurren e incluso, con el hecho de que fuera "natural" esperar los efectos luego de ocurrido u originado el evento. Esto significa que los llamados "desastres naturales" no son más que las consecuencias perniciosas de la ocurrencia de eventos asociados a fenómenos regulares o irregulares de la naturaleza, sean estos de tipo meteorológico, geotectónico o biológico (Alesch y Petak, 1996). No obstante, esta definición preliminar ha sido complementada por la literatura especializada a partir de la consideración de factores 'no naturales' (en el sentido utilizado hasta ahora) que contribuyen a desencadenar los desastres, entre estos, el estado social, político, económico y/o cultural de una población (BID, 2000; Blaikie et al., 1996; Dayton-Johnson, 2006; Dilley et al., 2005; Handmer y Dovers, 2007).

De acuerdo con lo anterior, los denominados "desastres naturales" serían el resultado de eventos de la naturaleza sumados al efecto de factores sociales, políticos, culturales y económicos, los que en definitiva incidirían en el nivel de vulnerabilidad de una determinada zona, región o país. En tal sentido, los desastres han llegado a ser entendidos como fenómenos eminentemente sociales (Lavell, 1993) ya que se concibe al evento natural como una condición necesaria, pero no suficiente para la ocurrencia de un desastre. Otras condiciones asociadas serían una estructura social, económica y/o política vulnerable (v.g. inequidad, calidad de la infraestructura, normas de construcción, niveles de asociatividad, diseño institucional de los organismos públicos, etc.). En tal sentido, los desastres vendrían 
a ser un componente revelador de un sistema social y político en un momento determinado (Clausen, 1994). Incluso, autores como Lewis (1977) han concebido los desastres no como acontecimientos singulares, sino como manifestaciones extremas de contextos cotidianos de existencia.

Considerando lo anterior, cabe destacar el hecho de que los efectos de un evento natural podrían ser "controlados" (atenuados o amplificados) mediante el uso de instrumentos de gestión y política, como por ejemplo, la planificación de los asentamientos humanos, la implementación de medidas de seguridad, planes de emergencia, sistemas de alerta, etc. Si bien los eventos que originan los desastres no pueden ser anticipados, y menos evitados, en principio sí pueden ser controlados sus efectos mediante el diseño y aplicación de efectivas políticas públicas de prevención (Comfort, Wisner et al., 1999; Charvériat 2000, Freeman et al., 2003; Kunreuthe, 2006, entre otros autores).

Así como un gran terremoto permite poner a prueba la calidad de las construcciones e infraestructura de la zona afectada, de manera análoga, los desastres constituyen instancias que otorgan a las ciencias sociales la oportunidad de estudiar un sistema social o político durante periodos de crisis, revelando y poniendo a prueba también instituciones, liderazgos y procesos básicos como la reacción de las organizaciones y personas y la articulación de información. De especial interés para la ciencia política resulta el estudio de la forma en que se toman (o no) las decisiones durante y después de una catástrofe:

crises make and break political careers, shake bureaucratic pecking orders and shape organizational destinies. Crises fix the spotlight on those who govern. Heroes and villains emerge with a speed and intensity quite unknown to 'politics as usual' (Handmer y Dovers, 2007: 43).

En tal sentido, sostenemos que el institucionalismo histórico, utilizado como enfoque, y el análisis de coyunturas críticas, utilizado como modelo, nos permiten analizar las crisis como momentos formativos en el desarrollo de la institucionalidad política, y llegar a algunas conclusiones acerca de la capacidad de esa institucionalidad de dar respuestas nuevas crisis y desafíos emergentes. 


\section{PolíticAs de CATÁstrofes}

La Organización de Naciones Unidas ha presentado indicaciones sobre políticas marco para del tratamiento de catástrofes derivadas de fenómenos naturales. En 2005, se celebró la Conferencia Mundial sobre la Reducción de los Desastres en la localidad japonesa de Hyogo, oportunidad en la que fue aprobado el "Marco de Acción para 2005-2015” que rige hoy la operación de estrategias en esta materia a nivel mundial. En términos generales, la Conferencia promovió un enfoque estratégico y sistemático sobre la reducción de la vulnerabilidad de las amenazas y los riesgos asociados, dando énfasis a los mecanismos por medio de los cuales robustecer la resiliencia de las comunidades frente a situaciones de catástrofes.

A su vez, Naciones Unidas detalla las definiciones de vulnerabilidad y amenaza o peligro. El primero de estos conceptos se refiere a las "condiciones determinadas por factores o procesos físicos, sociales, económicos o ambientales que aumentan la susceptibilidad y exposición de una comunidad al impacto de amenazas". Por otra parte, las amenazas comprenden

un evento físico potencialmente perjudicial, fenómeno o actividad humana que puede causar pérdida de vidas o lesiones, daños materiales, grave perturbación de la vida social y económica o degradación ambiental. Las amenazas incluyen condiciones latentes que pueden materializarse en el futuro. Pueden tener diferentes orígenes: natural (geológico, hidrometeorológico y biológico) o antrópico (degradación ambiental y amenazas tecnológicas). (ONU, 2006).

Naciones Unidas rescata, en este sentido, las experiencias de la "Estrategia de Yokohama”, aprobada en 1994, sobre la prevención, preparación y mitigación de los desastres naturales. Como resultado del balance del examen sobre los progresos obtenidos de aquella estrategia, se determinó la existencia de cinco falencias capitales: 1) gobernanza; 2) alerta temprana de los riesgos; 3) gestión de los conocimientos y educación; 4) reducción de factores de riesgo subyacentes; y 5) preparación para una respuesta eficaz y una recuperación efectiva (ONU, 2006). En función de estos resultados, la Conferencia adoptó los objetivos estratégicos de integración efectiva de la consideración de riesgos en las políticas y programas 
de desarrollo sostenido, creación de instituciones y mecanismos que fortalezcan la capacidad de resiliencia, en especial a nivel comunitario, y la incorporación de criterios de reducción del riesgo en fases de reconstrucción en las localidades damnificadas. De este modo, se estableció que el enfoque en materia de reducción de riesgos debía determinar al Estado como el principal responsable de las medidas contra el impacto de las catástrofes (ONU, 2006).

En concreto, las prioridades de acción definidas por Naciones Unidades se concentran en cinco puntos. En primer lugar, se plantea velar por que la reducción de los riesgos de desastre constituya una prioridad nacional y local dotada de una sólida base institucional de aplicación. Este principio contempla, primero, la formación de marcos institucionales y legislativos nacionales, que establezcan responsabilidades concretas, faciliten la coordinación y mantengan un diálogo entre el nivel nacional y regional. Segundo, la gestión de recursos, en relación con la evaluación de las necesidades de recursos humanos para la reducción de los riesgos, y la consignación de fondos para el diseño y la aplicación de programas de gestión de riegos a nivel nacional. Y tercero, la participación de la comunidad, en materia de fomentar la acción concertada y la colaboración estratégica de recursos voluntarios (ONU, 2006).

Por su parte, el Programa de Naciones Unidas para el Desarrollo (2004) señala la necesidad de analizar las políticas de prevención y tratamiento de los desastres desde una óptica contextual, en función de la máxima acerca de que los principales afectados por estas situaciones son los países de menor desarrollo y las personas de menores recursos. En este sentido, se reconoce que no solo los desastres naturales, vale decir, terremotos, ciclones tropicales, inundaciones y sequías, son trascendentales desde la óptica de las políticas en materia de devastación, sino que también son importantes factores de riesgos problemáticos como la violencia, los conflictos armados, las epidemias y la falta de gobernabilidad (PNUD, 2004: 9). La propuesta del PNUD es incorporar los riesgos de desastres en las políticas de desarrollo, combinando los criterios que rigen sus diseños. Desde esta perspectiva, los programas de gestión de riesgos debieran, por tanto, contemplar una esquematización del desarrollo sostenible como parte del trabajo prospectivo sobre los peligros, así como también un tipo de gestión compensatoria que comprenda una planificación de desarrollo que apunte a superar las condiciones de vulnerabilidad que propiciaron un impacto mayor de los efectos del desastre. 


\subsection{Toma de decisiones en medio de catástrofes naturales: reflexión sobre Nueva Orleans}

En agosto de 2005, el denominado huracán Katrina azotó las costas del sudeste de los Estados Unidos, con una de las mayores intensidades registradas en la historia norteamericana. Entre las principales localidades afectadas se menciona a Florida, Bahamas y Mississippi. Sin embargo, los mayores desastres se produjeron, puntualmente, en Nueva Orleans, al interior de Louisiana. Los efectos directos del huracán, sumados a la destrucción de los diques de contención provocaron la muerte de más de 1400 personas, cientos de miles de heridos, y decenas de miles de casa destruidas en esta última zona (Lousiana Department of Health and Hospitals, 2006).

Una de las principales aristas en torno al análisis del fenómeno, el manejo y los efectos del Katrina, tienen relación con la evaluación que se realiza sobre las políticas llevadas a cabo por el gobierno estadounidense. Hidalgo (2005), en este sentido, destaca el hecho de que el desastre natural que asoló a Nueva Orleans demostró que las catástrofes naturales pueden golpear de forma importante incluso a países fuertemente desarrollados, provocando profundos daños, lo que obligaría a repensar las políticas y los programas de prevención y tratamiento de estas situaciones.

Destacamos que cualquier investigación acerca de la forma en que se desarrollaron las consecuencias del huracán Katrina no debe pasar por alto el hecho de que se trató de un desastre previsto que ya había azotado las costas de Florida días antes. Las críticas sobre las pautas seguidas por el entonces presidente George W. Bush se sustentaron, en parte, en la inadecuada gestión de recursos y la demora en los tiempos de reacción, especialmente dado el aviso previo. Una crítica común apuntó a la considerada tardía y deficiente respuesta gubernamental a la crisis humanitaria derivada de Katrina, en especial sobre la insuficiencia de los servicios públicos. Otro cuestionamiento, de fundamentos más profundos, tuvo que ver con las características de la población residente en la zona del desastre. En Nueva Orleans el $27,9 \%$ vive por debajo de la línea de la pobreza, y el 67,3\% de sus habitantes son afroamericanos, composición que llevó a pensar en discriminación, marginación y una situación de vulnerabilidad mayor de las personas de menores recursos (GEAC - URJC, 2005). En conjunto, la gravedad del asunto condujo al 
cuestionamiento de Estados Unidos como potencial mundial en materia de labor de sus autoridades.

En definitiva, la respuesta efectiva demoró cuatro días sólo en lo que se refiere a actividades indirectamente vinculadas a la mejora de acceso humanitario y la cobertura de las personas que necesitaban asistencia. Más de 20 mil evacuados fueron llevados al estadio de fútbol Superdomo e incluso el Centro de Convenciones de Nueva Orleans fue adaptado como refugio.

Al igual que el huracán Katrina, el terremoto del 27/2 no solo afectó y devastó una gran extensión de territorio, sino que también dio origen a un amplio debate sobre el liderazgo de las autoridades de gobierno antes, durante y después de ambos desastres. Así como en EE.UU. fueron puestas en tela de juicio las acciones del presidente Bush, el Gobernador de Louisiana, el Alcalde de Nueva Orleans, el Secretario de Seguridad Interior y la autoridad Federal de Manejo de Emergencias, en Chile se cuestionó la respuesta de diversas autoridades políticas y militares, partiendo por la Presidenta de la época, por las acciones emprendidas -o no- así como por las decisiones tomadas -o no-. Incluso, por razones similares, como en el caso de Bush, acusado en su momento de haber sido "reacio" al envío de tropas al lugar de la catástrofe en New Orleans (Cohen et al., 2005).

Sin embargo los huracanes (y otros desastres naturales como inundaciones, tornados, etc.) poseen una característica que los hace ser cualitativamente distintos de los terremotos. Todos tienen un elemento de previsibilidad, mientras que los terremotos son imposibles de predecir, diferencia que se ha reconocido en la literatura especializada:

Finally, while hurricanes provide some lead time to prepare for the storm, earthquakes provide no such lead time, thereby making them even more frightening. Any preparations for earthquakes must be made before such an even strikes, whereas the lead time before a hurricanes gives people the sense that they 'can do something' to protect themselves, even if they have been lax in their preparations before the disasters (Birkland, 1997: 57-8).

Por esta razón, y para efectos del presente análisis, se estima que la catástrofe más parecida a un terremoto es un ataque terrorista. En ambos casos, la capacidad 
de reacción depende directamente de las medidas que se han tomado previo al incidente mismo, elementos como la inteligencia militar, planes de evacuación y rescate, y medidas de seguridad en sitios vulnerables, en el caso del terrorismo, y normas de construcción, oficinas de emergencia bien preparadas, con equipos científicos especializados, planes de control territorial y seguridad, en el caso de un terremoto.

\section{LAS COYUNTURAS CRÍTICAS Y EL 27F}

$\mathrm{Al}$ aplicar los principales elementos del institucionalismo histórico a los eventos del 27F, de debe comenzar el análisis por las instituciones que existían previo a terremoto. Antes de precisar las políticas específicas, podemos graficar el path de $27 \mathrm{~F}$ de la siguiente manera:

\section{Cuadro 2}

\section{Path Dependence y el 27/F}

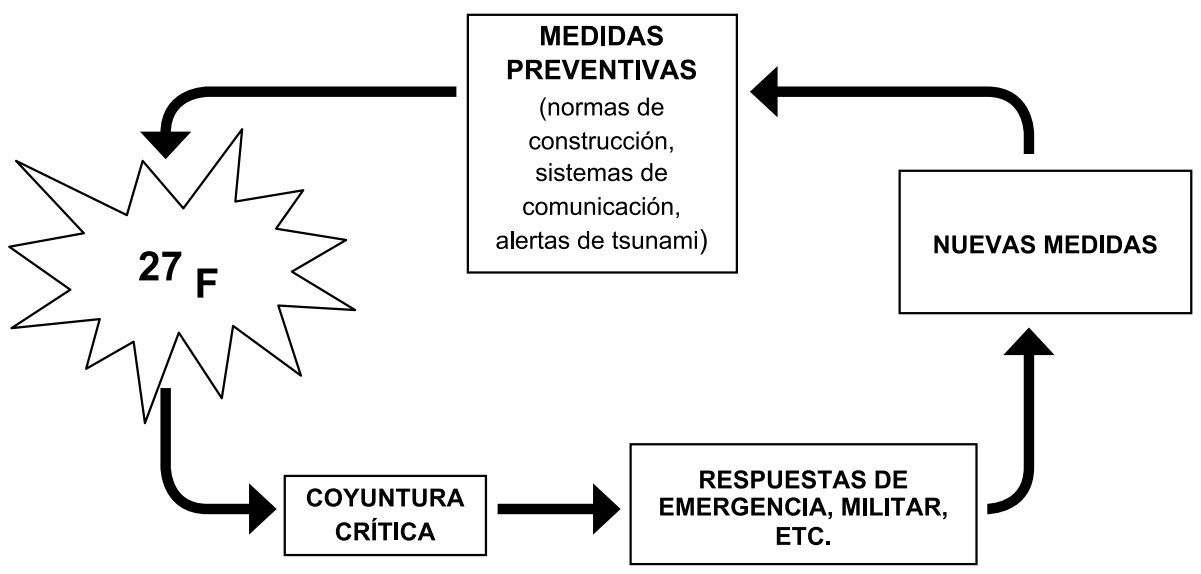

Fuente: Elaboración propia basado en Collier y Collier (1991).

Aplicando, entonces, el institucionalismo histórico a los eventos del 27F, el análisis comienza con el institucionalismo que existió previo al terremoto. Dentro de estas se encuentran las normas de construcción que fijan las capacidades de 
los edificios a resistir sismos (NCh433 de 1996, fortalecida con la NCh430 de 2008), planes reguladores de los municipios, y los planes e institucionalidad de emergencia, incluyendo la Oficina Nacional de Emergencia y el Servicio Hidrográfico y Oceanográfico de la Armada. Cada una de estas sería cuestionada en las semanas y meses después de $27 \mathrm{~F}$.

Uno de los elementos de la institucionalidad vigente más cuestionada sería el NCh433 y la NCh430, porque los edificios emblemáticos que evidenciaron daños fueron construidos en los años desde que esa norma entró en vigencia. El cuestionamiento a las normas de construcción vigentes se desarrolla a pesar de que la gran mayoría de los daños fueron sufridos por viviendas construidas de adobe y de albañilería sin refuerzo, construcciones no cubiertas por estas normativas, y buena parte de las muertes no fueron causadas por derrumbes, sino por el tsunami. En el caso de Santiago, un 14,4\% de los edificios sufrieron serios dańos pero solo un $0,3 \%$ se derrumbaron (mayores detalles ver la investigación de Ramírez, Fouillioux, Fossa, 2010).

Una investigación encontró que un problema recurrente de las construcciones nuevas se encontraba en los primeros pisos y los subsuelos, que, para permitir más estacionamiento y otros usos del suelo, cuentan con menos muros de corte y estos son más delgados (ver Ramírez, Schüler, Avendaño, 2010). En los meses siguientes al terremoto se revisan y se establecen nuevas normas de diseño (ver El Mercurio 07/10/2010). Esta revisión sigue fielmente el modelo temporal que nos indica el path dependency, manifestándose un régimen institucional previo al desastre, la puesta en evidencia de serias falencias, y el surgimiento paulatino de una nueva institucionalidad basada en el análisis de esas falencias.

En cuanto a la institucionalidad de preparación de emergencias, la Oficina $\mathrm{Na}$ cional de Emergencias contaba con una serie de planes e instrumentos antes del 27F, incluyendo el manual "Metodología básica para la elaboración de un plan comunal de prevención y de respuesta ante Tsunami” que, según informó el diario electrónico El Mostrador (02/08/2010), señalaba que:

deberá bastar la ocurrencia de un sismo local de gran intensidad, que impida a las personas mantenerse en pie, que haga caer muros, derrumbe torres y logre desplazar algunas casas de madera, para 
declarar la Alerta Roja y activar al Sistema de Protección Civil aplicando "el Plan de Emergencia en su fase de Evacuación hacia zonas seguras".

Dicho plan nunca se efectuó.

Al ocurrir el terremoto, durante ese día sábado, el gobierno de Michelle Bachelet, a través del Decreto Supremo No 150, declara zona de catástrofe las zonas afectadas. El día siguiente, con los Decretos No 152 y No 153, se declara un Estado de Excepción Constitucional de Catástrofe por Calamidad Pública en las regiones del Maule y Biobío. A pesar de estas medidas, las imágenes transmitidas por la televisión muestran una lenta reacción tanto en hacer llegar ayuda a los damnificados, como en la toma de control de las calles y el restablecimiento del orden público. Meses después se publicaría un video mostrando la descoordinación que existió en las oficinas de la ONEMI en las primeras horas del 27 de febrero ${ }^{1}$.

Una vez asumido en el poder, el gobierno de Sebastián Piñera diseñó el Plan de Reconstrucción "Chile Unido Reconstruye Mejor" (2010), a cargo del Ministerio de Vivienda y Urbanismo, el cual comprendió 2.500 millones de dólares para la reconstrucción de 220 mil viviendas en 900 localidades y 45 ciudades. En lo específico, el Plan de Reconstrucción del MINVU fue coordinado por un grupo de trabajo especializado, encabezado por la Ministra y el Subsecretario de la materia y conformado de acuerdo a tres escalas de trabajo:

a) Vivienda: pensado, en lo sustancial, para la entrega de subsidios para la reedificación de viviendas dañadas o la obtención de nuevos inmuebles;

b) Barrios: su objetivo es dar una solución definitiva a los problemas habitacionales en relación con el grupo de trabajo Aldeas y Condominios sociales; $y$

c) Ciudad y Territorio: referido a la modernización de los Planes reguladores en función de su adaptación a los riesgos, así como también sobre la recuperación y fortalecimiento de las zonas y edificios

1 Dicho video se encuentra disponible en: http://latercera.com/contenido/680_257188_9.shtml 
patrimoniales, todo en el marco de los denominados Planes Maestros (Gobierno de Chile, 2010: 14).

A la vez, el presidente anunció un recorte de $5 \%$ en los presupuestos de cada ministerio, y habló de un plan de austeridad parecido a lo que le correspondería a una "economía de guerra". Una reforma tributaria subiría el impuesto a las empresas de un $17 \%$ a un $20 \%$ durante el 2011, reduciéndolo durante los dos años siguientes para volver al $17 \%$ en 2013 . Un nuevo "royalty" -que en estricto rigor no corresponde al concepto de royalty, normalmente usado para referirse a una tasa adicional de impuesto por sobre las utilidades y usado para compensar los efectos directos de una industria en particular o financiar alternativas para aquellas industrias que no son viables en el largo plazo- terminará siendo un alza a las utilidades operacionales.

Esto es acompañado por medidas paliativas más inmediatas, incluyendo las Leyes $20.436,20.438,30.440,20.444,20.451,20.446$ y otras, destinadas a modificar y flexibilizar leyes y normas de la vida cotidiana (plazos para procesos judiciales, cambios en subvenciones a la educación, incentivos a donaciones, etc.). A la vez, se establecen una serie de medidas administrativas, como la creación de un Comité de Emergencia (Decreto Supremo 350) o la delegación de facultades presidenciales a autoridades regionales de defensa (Decreto Exento 861). Sin embargo, la premura también provoca errores, como el que llegó a la luz cuando la Contraloría General de la República diera a saber irregularidades por parte del Comité de Emergencia en la compra de mediaguas, incluyendo el sobrepago de millones de pesos o la confección de contratos con empresas que no contaban con la capacidad de cumplir con ellos ${ }^{3}$.

Una de las medidas más esperadas y aún no realizadas es una reforma a la Oficina Nacional de Emergencia. En su discurso del 21 de mayo de 2010, el presidente Piñera anunció la creación de una Agencia Nacional de Emergencia. En julio del mismo año el Subsecretario de Interior, Rodrigo Ubilla, declaró que "la Onemi

2 http://www.nacion.com/2010-03-20/Mundo/UltimaHora/Mundo2309617.aspx

3 http://www.emol.com/noticias/nacional/detalle/detallenoticias.asp?idnoticia=456723\&utm source $=$ detalleNoticia\&utm_medium $=$ relacionados\&utm_campaign $=$ Oposici $\%$ F3n $\% 20$ exige $\% 20$ sanciones $\% 20$ por $\% 20$ informe $\% 20$ de $\% 20$ Contralor $\%$ EDa $\% 20$ que $\% 20$ acredit $\%$ F3\%20sobrepago $\% 20 \mathrm{de} \% 20$ mediaguas 
es uno de los aspectos más relevantes en el marco de la modernización del Ministerio del Interior" ${ }^{\text {. }}$ A la fecha, sin embargo, no se ha presentado un proyecto de ley al respecto.

\section{CONCLUSIONES}

La aplicación del institucionalismo histórico a los eventos del 27F y los días siguientes nos permite identificar algunas fallas, pero también éxitos, importantes en la reacción al terremoto. Entre las primeras se encuentra primordialmente el tema de las comunicaciones.

Entre los éxitos, se destacan las normas de construcción que, si bien sufrieron un período de debilitamiento, en general resultaron en la edificación de estructuras sólidas y capaces de resistir terremotos de gran envergadura. En Santiago, e incluso en Concepción, la gran mayoría de edificios, aunque haya sufrido daños mayores, resistieron lo suficiente como para permitir que las personas no quedaran atrapadas en los escombros.

Si bien las medidas tomadas en función de la reconstrucción han sido criticadas, los esfuerzos para corregir algunas de las políticas públicas que no funcionaron adecuadamente en el desastre del 27F han sido más exitosos. Medidas como las señaladas anteriormente dan fe de un reconocimiento de que el statu quo no ofrecía las garantías suficientes para permitir una reacción eficiente y efectiva. Por cierto, este tipo de reconocimiento se hizo más fácil en un contexto político en que la responsabilidad por las faltas se podía pasar a un gobierno anterior.

En esto, como en tantos otros elementos, llama la atención las similitudes con el ataque terrorista ocurrido en Nueva York, Washington y Pennsylvania el 11 de septiembre de 2001, conocido como 9-11.

4 http://informa.gob.cl/comunicados-archivo/subsecretario-ubilla-onemi-constituye-uno-delos-aspectos-mas-relevantes-en-la-modernizacion-del-min/ 


\section{Cuadro 3}

\section{Path Dependence y el 11/S}

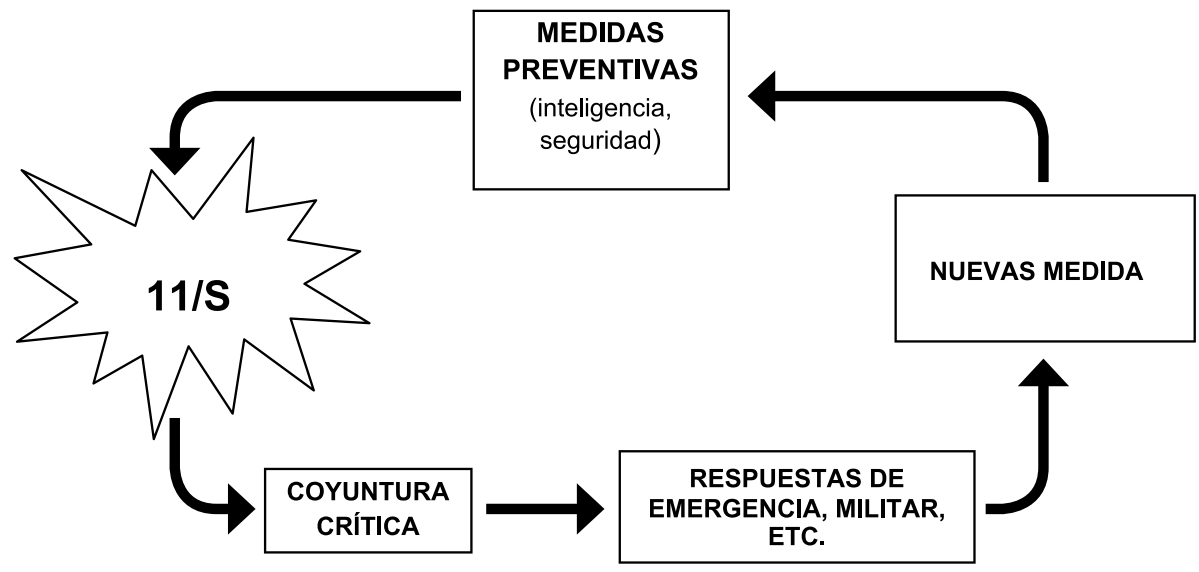

Fuente: Elaboración propia basado en Collier y Collier (1991).

Desastres naturales como los huracanes se pueden anunciar con algo de anticipación. Los ataques terroristas, al igual que los terremotos -si bien caen dentro de un rango de probabilidades- ocurren sin advertencias.

Este aspecto le otorga una especial importancia a las políticas públicas de preparación, para maximizar las posibilidades tanto de detección como de reacción.

Segundo, como demuestra el gráfico $\mathrm{N}^{\circ} 2$ en el artículo de Oscar Landerretche (pág. 47 de este volumen) en los casos de los terremotos, como en ataques terroristas, el punto de mayor temor -inmediatamente después del evento- coincide con el momento en que es menos probable que ocurra otro incidente. Sin embargo, en la medida que pasa el tiempo, va subiendo la probabilidad de otro ataque/ terremoto, pero se reduce la ansiedad. El desafío para las políticas públicas es manejar los niveles de temor y ansiedad, de manera de no crear pánico, pero de permitir un nivel de conciencia pública suficiente como para permitir la mantención de políticas públicas de preparación y reacción (que muchas veces implican altos costos económicos). 
Una tercera similitud es que, al verse obligados a reaccionar frente a las fallas de reacción, y en el contexto de un sentido de 'emergencia' o 'unidad nacional', los gobiernos suelen incluir en sus propuestas políticas elementos que tienen poco o nada que ver con la emergencia misma, o, en el espíritu de 'unidad nacional', agregar políticas que planeaban implementar de todas maneras, como, por ejemplo, la invasión de Irak, en el caso de Estados Unidos, o los recortes a los gastos impuestos por el plan de austeridad firmado por el presidente Piñera en marzo de 2010.

Estas y otras medidas dan fe de cómo eventos -naturales o no- obligan a los tomadores de decisiones a optar por políticas que, en el mediano y largo plazo, no solamente inciden en la reacción a esos eventos mismos, sino que van creando nuevas realidades. Si se obtiene el apoyo político necesario para legitimar estas decisiones, las políticas se van institucionalizando, convirtiéndose, a la vez, en esa nueva realidad. Por eso es de suma importancia hacer un seguimiento de las decisiones tomadas, pues la nueva institucionalidad será la que determinará la capacidad de respuesta en futuros eventos críticos.

\section{REFERENCIAS BIBLIOGRÁFICAS}

Alesch, Daniel J. y Petak, William J. (1996). “The Politics and Economics of Earthquake Hazard Mitigation. Unreinforced Masonry Buildings in Southern California”. Monograph No43, Program on Environment and Behavior, University of Colorado.

Araújo, Antonio (2007). "Path dependency en los Estudios Históricos Comparados". XXIV SimposioNacional de Historia, Asociación Nacional de Historia.

Banco Interamericano de Desarrollo. (2000). El desafí de los desastres naturales en América Latina y el Caribe. Washington: Banco Interamericano de Desarrollo.

Birkland, Thomas. (1997). After disaster. Agenda setting, public policy and focusing events. Washington: Georgetown University Press.

Blaikie, P., Cannon, T., Davis, I., Wisner, B. (1996). Vulnerabilidad. El entorno social, politico y económico de los desastres. Colombia: Red de Estudios Sociales en Prevención de Desastres en América Latina. 
Charvériat, C. (2000). Natural Disaster Risk in Latin America and the Caribbean. Washington, D.C: InterAmerican Development Bank.

Clausen, Lars; Conlon, Paul; Jager, Wieland; Metreveli, Stephan. (1978). "New Aspects of the Sociology of Disasters: A Theoretical Note". Mass Emergencies and Disasters Vol. 3, No. 1, pp. 61-65.

Collier, Ruth y David Collier. (1991). Shaping the political arena. Critical juncture, the labor movement, and regime dynamics in Latin America. New Jersey: Princeton University Press.

Comfort, L., Wisner, B., et al. (1999). "Reframing disaster policy: the global evolution of vulnerable communities". Evironmental Hazards Vol. 1, No1, pp. 39-44.

Dayton-Johnson, J. (2006). "Natural Disaster and Vulnerability”. OECD Development Centre Policy Brief, No 29.

Dilley, M., Chen, R.S., Deichmann, U., LernerLam, A.L., Arnold, M., Agwe, J., Buys, P., Kjekstad, O., Lyon, B. y Yetman, G. (2005). Natural Disaster Hotspots. A Global Risk Analysis. Disaster Risk Management Series. Washington: The International Bank for Reconstruction and Development, World Bank and Columbia University.

Freeman, P. et al. (2003). Disaster Risk Management. Integration and Regional Programs. Washington: Department Regional Policy Dialogue.

GEAC, URJC (2005). En el ojo del huracán: la imagen mediática de EEUU tras el paso del Katrina. Análisis de la prensa de referencia de Brasil, Gran Bretaña, Italia y España. Real Instituto Elcano de Estudios Internacionales y Estratégicos, Área: Imagen exterior de España y Opinión Pública - DT No 51 .

Gobierno de Chile (2010) Chile Unido Reconstruye Mejor. Santiago: Ministerio de Vivienda y Urbanismo.

Hall, Peter A. y Rosemary C. R. Taylor (1996). "Political Science and the Three New Institutionalisms", Political Studies Vol. 44, No 5, pp. 936-57.

Handmer, J. W. y Dovers, S. (2007). The handbook of disaster and emergency policies and institutions. London: Earthscan.

Kunreuther, H. (2006). "Disaster Mitigation and Insurance: Learning from Katrina”. The Annals of the American Academy of Political and Social Science, Vol. 604, No 1, pp .208-227.

Lavell, Th. A. (1993). "Ciencias Sociales y desastres naturales en América Latina: un encuentro inconcluso”. EURE, Vol. XIX, N 58, pp. 73-84.

Lewis, James. (1977). "Some aspects of disaster research". Disasters, Vol. 1, No 3, pp. 241-244. 
Organización de Naciones Unidas (2006). Marco de Acción de Hyogo para 2005-2015: Aumento de la resiliencia de las naciones y comunidades ante los desastres. Kobe: Conferencia Mundial sobre la Reducción de los Desastres, Estrategia Internacional para la Reducción de Desastres.

Peters, Guy (2005). Institutional Theory in Political Science: The 'New Institutionalism'. New York y London: Continuum.

Pierson, Paul y Skocpol, Theda. (2008). "El institucionalismo histórico en la ciencia política contemporánea”. Revista Uruguaya de Ciencia Política, Vol. 17, No 1, pp. 7-38.

Pierson, Paul. (2000). "Increasing Returns, Path Dependence, and the Study of Politics", American Political Science Review Vol. 94, No 2, pp. 251-67.

Programa de las Naciones Unidas para el Desarrollo (2004). Las reducciones de riesgos de desastres. Un desafio para el desarrollo. PNUD, Dirección de Prevención de Crisis y de Recuperación.

Sánchez, Manuel (2004). "Estudio comparado de Path Dependence del Estado de Bienestar en los casos de USA, Suecia y España”. Revista de Estudios Políticos Nueva Época No 124, pp. $95-128$.

Skocpol, Theda. (1979). States and Social Revolutions: A Comparative Analysis of France, Russia, and China. Cambridge and New York: Cambridge University Press.

\section{Paginas web:}

Cohen, Morris A., Lawrence G. Hrebiniak, y Robert E. Mittelstaedt Jr. (2005). "A Month after Katrina: Lessons from Leadership Failures." Interview. Wharton Risk Management and Decision Processes Center. Wharton University of Pennsylvania. En: http://knowledge. wharton.upenn.edu/article.cfm?articleid=1289.

http://informa.gob.cl/comunicados-archivo/subsecretario-ubilla-onemi-constituye-uno-de-losaspectos-mas-relevantes-en-la-modernizacion-del-min/

http://www.nacion.com/2010-03-20/Mundo/UltimaHora/Mundo2309617.aspx

Louisiana Department of Health and Hospitals (2006). Reports of Missing and Deceased. www. dhh.louisiana.gov/offices/page. asp?ID=192\&Detail $=5248$

Pedro Ramírez, Matías Fouillioux y Lissette Fossa, A seis meses del terremoto: Los cambios en normas de la construcción que impulsa la ministra Matte http://ciperchile.cl/2010/08/26/aseis-meses-del-terremoto-los-cambios-a-las-normas-de-la-construccion-que-impulsa-laministra-matte/ 
Pedro Ramírez, Úrsula Schüler y Felipe Avendaño Estudio de edificio de Penta revela falla que se repite en modernas torres afectadas por el terremoto http://ciperchile.cl/2010/04/07/estudio-de-edificio-de-penta-revela-la-falla-estructural-que-se-repite-en-las-modernas-torresafectadas-por-el-terremoto/

www.elmostrador.cl

www.emol.com.

www.lasegunda.com

www.latercera.com

\section{Documentos:}

Norma Chilena 433 - 1996 "Diseño sísmico de edificios”.

Norma Chilena 430 - 2008 "Hormigón Armado. Requisitos de Diseño y Cálculo". 